PROCEEDINGS OF THE

AMERICAN MATHEMATICAL SOCIETY

Volume 144, Number 4, April 2016, Pages 1523-1533

http://dx.doi.org/10.1090/proc/12956

Article electronically published on December 22, 2015

\title{
ON THE SECOND ORDER DERIVATIVES OF SOLUTIONS OF A SPECIAL ISAACS EQUATION
}

\author{
JAY KOVATS
}

(Communicated by Joachim Krieger)

\begin{abstract}
In this paper, we investigate continuity and integrability properties of the second order derivatives of viscosity solutions of a certain uniformly elliptic Isaacs equation. We give sufficient and necessary conditions for continuity of the second order derivatives and give sufficient conditions for interior $W^{2, p}$ regularity, $1<p<\infty$.
\end{abstract}

\section{INTRODUCTION}

In this paper, we examine continuity and integrability properties of the second order derivatives of viscosity solutions $u \in C(D)$ of the uniformly elliptic Isaacs equation

$$
F\left(u_{x x}\right):=\Delta u+\left(u_{x^{1} x^{1}}\right)_{+}-\left(u_{x^{2} x^{2}}\right)_{-}=0 \quad \text { in } D,
$$

where for $a \in \mathbb{R}, a_{+}:=\max \{a, 0\}, a_{-}:=-\min \{a, 0\}$. Here, $D$ is a domain in $\mathbb{R}^{d}$ with $d \geq 3$, and $u_{x x}=\left(u_{x^{i} x^{j}}\right)$ denotes the Hessian matrix of $u$. We recall that the second order equation $F\left(u_{x x}\right)=0$ is uniformly elliptic if there exist constants $0<\lambda \leq \Lambda$ (called ellipticity constants) such that $\forall m \in \mathcal{S}_{d}$ (the space of real symmetric $d \times d$ matrices)

$$
\lambda\|n\| \leq F(m+n)-F(m) \leq \Lambda\|n\| \quad \forall 0 \leq n \in \mathcal{S}_{d},
$$

where for $m \in \mathcal{S}_{d},\|m\|:=\sup _{e \in \mathbb{R}^{d},|e|=1}|m e|$. A uniformly elliptic Isaacs equation, in its simplest form, is a second order pde of the form

$$
F\left(u_{x x}\right):=\max _{y \in Y} \min _{z \in Z} \operatorname{tr}\left[a(y, z) u_{x x}\right]=0
$$

(or with $\min _{z \in Z} \max _{y \in Y}$ ). Here, tr denotes trace, $Y, Z$ are compact sets in Euclidean space, $a(y, z)$ is a $d \times d$ matrix defined on $Y \times Z$ and there exist constants $0<\lambda \leq \Lambda$, for which $\lambda I_{d} \leq a(y, z) \leq \Lambda I_{d}$, for all $y, z$. From the elementary inequalities

$$
\inf _{y, z}\left(f_{y, z}-g_{y, z}\right) \leq \sup _{y} \inf _{z} f_{y, z}-\sup _{y} \inf _{z} g_{y, z} \leq \sup _{y, z}\left(f_{y, z}-g_{y, z}\right),
$$

Received by the editors September 30, 2014.

2010 Mathematics Subject Classification. Primary 35B65, 35J60, 49N60, 49N70; Secondary $35 \mathrm{~J} 60$.

(C)2015 American Mathematical Society 
it follows that equation (0.3) is uniformly elliptic with ellipticity constants $\lambda, \Lambda d$. Since our pde in (0.1) can be written as

$F\left(u_{x x}\right):=\max _{1 \leq y \leq 2} \min _{1 \leq z \leq 2} \operatorname{tr}\left[a(y, z) u_{x x}\right]=0, \quad$ where $a(y, z)=\left(\begin{array}{cccc}y & & & \\ & z & & \\ & 1 & \\ & & \ddots & \\ & & & \\ & & \end{array}\right), y, z \in[1,2]$,

it follows that equation (0.1) is uniformly elliptic with ellipticity constants $1,2 d$.

Isaacs equations are of interest from both a probabilistic and a differential equations standpoint. From the probabilistic viewpoint, an Isaacs equation is the dynamic programming equation of a zero-sum, stochastic differential game between two players, one attempting to maximize a given functional and the other attempting to minimize the functional (see [FN93, FS89, Kov09, Kry14a, Kry14b, Nis88). From a pde standpoint, the Isaacs equation is the prototypical example of a uniformly elliptic pde which is neither convex nor concave in $u_{x x}$. The $C^{2, \alpha}$ regularity theory for elliptic equations was based on the assumption that in the uniformly elliptic equation $F\left(u_{x x}\right)=0$, the operator $F=F(m)$ was either convex or concave in $m$. The example in [NV07] showed that the classical solvability of the Dirichlet problem with continuous boundary values is not guaranteed if this condition fails. Moreover, the conclusion of the Evans-Krylov theorem (see Eva82, Kry83) may fail if this condition is dropped (see NV08). Hence, regularity results for solutions of nonconvex equations are of interest. The $C^{2, \alpha}$ regularity of solutions to certain classes of nonconvex uniformly elliptic equations of the form $F\left(u_{x x}\right)=0$ has been proved in a number of papers (see e.g., CC03, CY00, Yua01).

It is well known (see [sh89, CC95) that if $F$ is uniformly elliptic and $D$ is a smooth domain, then for arbitrary $g \in C(\bar{D})$, there exists a unique viscosity solution $u \in C(\bar{D})$ of the Dirichlet problem

$$
\left\{\begin{aligned}
F\left(u_{x x}\right)=0 & \text { in } D \\
u=g & \text { on } \partial D .
\end{aligned}\right.
$$

It is also well known that for general bounded domains $D \subset \mathbb{R}^{d}$, if $u \in C(D)$ is a viscosity solution of $F\left(u_{x x}\right)=0$ in $D$, then the second derivatives of $u$ exist almost everywhere in $D$ (see [Tru89]) and belong to $L_{l o c}^{\delta}(D)$ for some small $\delta=\delta(d, \lambda, \Lambda)>$ 0 (see [Lin86]). In particular, viscosity solutions of Isaacs equation (0.3) belong to $W_{l o c}^{2, \delta}(D)$ and satisfy the following interior estimate: $\forall B_{r}:=B_{r}(y) \subset \subset D$

$$
\left\|u_{x x}\right\|_{L^{\delta}\left(B_{r / 2}\right)} \leq N(d, \lambda, \Lambda) r^{-2+\frac{d}{\delta}} \sup _{B_{r}}|u| .
$$

But other than locally belonging to $L^{\delta}$, little is known about the second order derivatives of viscosity solutions of $F\left(u_{x x}\right)=0$.

The example in NV08 tells us that in general, one cannot a priori expect solutions of $F\left(u_{x x}\right)=0$ to be locally $W^{2, \infty}$, or even $W^{2, p}$ for $p$ sufficiently large. However, the pde in NV08 was a Hessian equation, not an Isaacs equation. The $W^{2, p}$ regularity of viscosity solutions of Isaacs equations with compact control sets $Y, Z$ (an important feature for applications) is an open question. It is well known (see CC03] that any uniformly elliptic operator $F(m)$ can be shown to be of $\mathrm{min} / \mathrm{max}$ type, though not necessarily satisfying the compactness condition of the control sets following (0.3). In particular, although the singular solution in NV11 was shown to satisfy a min/max equation in $B_{1} \subset \mathbb{R}^{12}$, it was not an Isaacs equation satisfying the conditions of (0.3). It is reasonable to expect that certain special cases of such 
equations may fare better. The Isaacs equations with finite (or more generally, compact in Euclidean space) control sets $Y, Z$ is an interesting and important case to consider.

In Kov12, we proved the $W^{2, p}$ interior regularity $(0<p<\infty)$ of a class of Isaacs equations (which includes (0.1)), under the assumption that solutions were $C^{2}$. In the present paper, we investigate regularity questions for viscosity solutions of equation (0.1) without this assumption.

The Isaacs equation $\Delta u+\left(u_{x^{1} x^{1}}\right)_{+}-\left(u_{x^{2} x^{2}}\right)_{-}=0$ is interesting for a number of reasons. First, in dimension $d=2$, the pde is equivalent to Laplace's equation $\Delta u=0$. Second, it is an example of a pde in which the operator $F=F(m)$ satisfies the minmax principle: for any $m \in \mathcal{S}_{d}$,

$$
\max _{y \in Y} \min _{z \in Z} \operatorname{tr}[a(y, z) m]=F(m)=\min _{z \in Z} \max _{y \in Y} \operatorname{tr}[a(y, z) m] .
$$

The min-max principle is an important property in stochastic differential games (see [Roc70, Nis88, Kov09]). Note the extrema over [1,2] are really extrema over $\{1,2\}$ and $(0.1)$ can be rewritten as

$$
\begin{aligned}
0 & =F\left(u_{x x}\right) \\
& =\max \left\{\Delta u+u_{x^{1} x^{1}}-\left(u_{x^{2} x^{2}}\right)_{-}, \Delta u-\left(u_{x^{2} x^{2}}\right)_{-}\right\}:=\max \left\{F^{+}\left(u_{x x}\right), F^{-}\left(u_{x x}\right)\right\}
\end{aligned}
$$

$$
=\min \left\{\Delta u+\left(u_{x^{1} x^{1}}\right)_{+}+u_{x^{2} x^{2}}, \Delta u+\left(u_{x^{1} x^{1}}\right)_{+}\right\}:=\min \left\{G^{+}\left(u_{x x}\right), G^{-}\left(u_{x x}\right)\right\},
$$

where $F^{+}, F^{-}$are concave, $G^{+}, G^{-}$are convex, and all are uniformly elliptic (with the same ellipticity constants $1,2 d)$. If say, either $F^{+}\left(u_{x x}\right)=0$ in $O$ or $G^{+}\left(u_{x x}\right)=0$ in $O$, where $O \subset D$ is any open set, then, by the Evans-Krylov theorem, $u \in$ $C_{l o c}^{2, \alpha}(O)$. A corresponding statement holds for $F^{-}, G^{-}$(see Lemma 2.1 below).

Finally, the pde (0.1) is of interest because of its simplicity. Not only does $F$ depend only on the second order derivatives of $u$, it depends only on the pure second order derivatives, i.e $F\left(u_{x x}\right)=F\left(u_{x^{1} x^{1}}, u_{x^{2} x^{2}}, \ldots, u_{x^{d} x^{d}}\right)$. Despite its simplicity, it is not of the form to which some recent regularity results (for nonconcave equations) apply (see [CC03, [CY00], [NV11]). For example, equation (0.1) is not expressible as the minimum of two operators, where one is convex and the other concave (see (0.6) above and (1.9) in [CC03]). Furthermore, the operator $F(m)$ does not have convex level sets (CY00), nor is it of Hessian type NV11. See example 1 in Kov12 for a more detailed discussion.

In Kov12 we proved that if $u \in C^{2}(D)$ is a solution of the Isaacs equation

$$
F\left(u_{x x}\right):=\max _{y \in Y} \min _{z \in Z} \operatorname{tr}\left[a(y, z) u_{x x}\right]=0 \quad \text { in a domain } D \subset \mathbb{R}^{d},
$$

where $Y, Z$ are compact sets in Euclidean space, the control matrix $a(y, z)$ is diagonal, separable, and $\lambda I_{d} \leq a(y, z) \leq \Lambda I_{d}$, then $u \in W_{l o c}^{2, p}(D), \forall 0<p<\infty$ and $\forall B_{r}:=B_{r}(y) \subset \subset D$,

$$
\left\|u_{x x}\right\|_{L^{p}\left(B_{r / 2}\right)} \leq N(d, p, \lambda, \Lambda) r^{-2+\frac{d}{p}} \sup _{B_{r}}|u| .
$$

In particular, $C^{2}(D)$ solutions of $\Delta u+\left(u_{x^{1} x^{1}}\right)_{+}-\left(u_{x^{2} x^{2}}\right)_{-}=0$ belong to $W_{l o c}^{2, p}(D)$, $\forall 0<p<\infty$, and satisfy interior estimate $(0.7)$.

In $\S 2$ of the present paper, we examine continuity and $p$-integrabilty properties of second order derivatives of solutions of (0.1) without the assumption that $u \in C^{2}$. In $\S 1$, we prove the $W^{2, p}$ regularity for $C^{2}$ solutions of (0.1). Although this result 
is proved in Kov12, we include the proof here, since some of the arguments will be used later in our proof of Theorem 2. It is interesting to note the contrast between the techniques used in the $C^{2}$ setting and those used in the viscosity solution setting. In particular, the proof of Theorem 1 relies on the fact that the sets $D^{+}:=\left\{D: u_{x^{1} x^{1}}>0\right\}$ and $D^{-}:=\left\{D: u_{x^{2} x^{2}}>0\right\}$ are open, assumptions that cannot be made for viscosity solutions.

\section{1. $W^{2, p}$ REgularity For $C^{2}$ SOlutions of $\Delta u+\left(u_{x^{1} x^{1}}\right)_{+}-\left(u_{x^{2} x^{2}}\right)_{-}=0$}

In this section, we prove that $C^{2}(D)$ solutions of (0.1) satisfy interior $W^{2, p}$ estimate $(0.5)$ for all $0<p<\infty$. Before doing so, we introduce some notation. Observe that any $m \in \mathcal{S}_{d}$ can be written as $m=m^{+}-m^{-}$, where $m^{+}, m^{-} \geq 0$ and $m^{+} m^{-}=0$. For $0<\lambda \leq \Lambda$ and $m \in \mathcal{S}_{d}$, we define Pucci's extremal operators $\mathcal{M}^{+}$and $\mathcal{M}^{-}$by

$\mathcal{M}^{+}(m, \lambda, \Lambda)=\Lambda \operatorname{tr}\left(m^{+}\right)-\lambda \operatorname{tr}\left(m^{-}\right), \quad \mathcal{M}^{-}(m, \lambda, \Lambda)=\lambda \operatorname{tr}\left(m^{+}\right)-\Lambda \operatorname{tr}\left(m^{-}\right)$.

For an open set $D \subset \mathbb{R}^{d}$, and $f \in C(D), \underline{S}(\lambda, \Lambda, f)$ is defined to be the set of $u \in$ $C(D)$ such that $\mathcal{M}^{+}\left(u_{x x}, \lambda, \Lambda\right) \geq f$ in the viscosity sense in $D$. Similarly, $\bar{S}(\lambda, \Lambda, f)$ denotes the set of $u \in C(D)$ such that $\mathcal{M}^{-}\left(u_{x x}, \lambda, \Lambda\right) \leq f$ in the viscosity sense in $D$. Functions in $\underline{S}$ or $\bar{S}$ are called subsolutions or supersolutions, respectively. (For more details, see $\S 2.2$ of [CC95].)

Theorem 1. Let $D$ be a domain in $\mathbb{R}^{d}$ and let $u \in C^{2}(D)$ satisfy $F\left(u_{x x}\right):=\Delta u+$ $\left(u_{x^{1} x^{1}}\right)_{+}-\left(u_{x^{2} x^{2}}\right)_{-}=0$ in $D$. Then $\forall B_{r}:=B_{r}\left(x_{0}\right) \subset \subset D$ and any $0<p<\infty$,

$$
\left\|u_{x x}\right\|_{L^{p}\left(B_{r / 2}\right)} \leq N(d, p) r^{-2+\frac{d}{p}} \sup _{B_{r}}|u| .
$$

We first prove the following subsolution lemma.

Lemma 1.1. Under the above assumptions, both $\left(u_{x^{1} x^{1}}\right)_{+}$and $\left(u_{x^{2} x^{2}}\right)_{-}$belong to $\underline{S}(0):=\underline{S}\left(\frac{1}{d}, 2 d, 0\right)$ in $D$.

Proof. Define $D^{+}=\left\{x \in D: u_{x^{1} x^{1}}(x)>0\right\}$, and for $h>0, D_{h}^{+}=\left\{x \in D^{+}\right.$: $\left.\operatorname{dist}\left(x, \partial D^{+}\right)>h\right\}$. Note that in $D^{+}, u$ satisfies $F^{+}\left(u_{x x}\right)(x):=\Delta u(x)+u_{x^{1} x^{1}}(x)-$ $\left(u_{x^{2} x^{2}}(x)\right)_{-}=0$. Fix $x \in D_{h}^{+}$and set $w^{h}(x)=u\left(x+h e_{1}\right)+u\left(x-h e_{1}\right)-2 u(x)$. Since $F^{+}\left(u_{x x}\right)\left(x \pm h e_{1}\right)=0$ in $D_{h}^{+}$, setting these 3 equations $=0$ and using $a_{-}+b_{-}-2 c_{-} \geq$ $-(a+b-2 c)+$ yields

$$
\begin{aligned}
0 & =\Delta w^{h}(x)+w_{x^{1} x^{1}}^{h}(x)-\left\{\left[u_{x^{2} x^{2}}\left(x+h e_{1}\right)\right]_{-}+\left[u_{x^{2} x^{2}}\left(x-h e_{1}\right)\right]_{-}-2\left[u_{x^{2} x^{2}}(x)\right]_{-}\right\} \\
& \leq \Delta w^{h}(x)+w_{x^{1} x^{1}}^{h}(x)+\left(w_{x^{2} x^{2}}^{h}(x)\right)_{+} .
\end{aligned}
$$

That is, in $D_{h}^{+}, w^{h}$ is a viscosity subsolution of the uniformly elliptic equation $\Delta v+v_{x^{1} x^{1}}+\left(v_{x^{2} x^{2}}\right)_{+}=0$, with the same ellipticity constants $1,2 d$. Thus $w^{h}$ and hence $\Delta_{h, e_{1}}^{2} u=\frac{w^{h}}{h^{2}} \in \underline{S}(0)$ in $D_{h}^{+}$. Since $u \in C^{2}(D)$ and $\underline{S}(0)$ is closed, $u_{x_{1} x_{1}} \in \underline{S}(0)$ in $D^{+}$, from which it follows that $\left(u_{x^{1} x^{1}}\right)_{+} \in \underline{S}(0)$ in $D$. Here we are using the fact that if $v \in C(D)$ and if $v \in \underline{S}(0)=\underline{S}(\lambda, \Lambda, 0)$ in $D^{+}:=\{x \in D: v(x)>0\}$, then $v_{+} \in \underline{S}(0)$ in $D$. 
To see that $\left(u_{x^{2} x^{2}}\right)_{-} \in \underline{S}(0)$ in $D$, define $D^{-}=\left\{x \in D: u_{x^{2} x^{2}}(x)<0\right\}$, and for $h>0, D_{h}^{-}=\left\{x \in D^{-}: \operatorname{dist}\left(x, \partial D^{-}\right)>h\right\}$. In $D^{-}, u$ satisfies $G^{+}\left(u_{x x}\right)(x):=$ $\Delta u(x)+\left(u_{x^{1} x^{1}}(x)\right)_{+}+u_{x^{2} x^{2}}(x)=0$. Fix $x \in D_{h}^{-}$and set $w^{h}(x)=u\left(x+h e_{2}\right)+$ $u\left(x-h e_{2}\right)-2 u(x)$. Since $G^{+}\left(u_{x x}\right)\left(x \pm h e_{2}\right)=0$ in $D_{h}^{-}$, setting these 3 equations $=0$ and using $a_{+}+b_{+}-2 c_{+} \geq-(a+b-2 c)_{-}$yields

$$
\begin{aligned}
0 & =\Delta w^{h}(x)+\left[u_{x^{1} x^{1}}\left(x+h e_{2}\right)\right]_{+}+\left[u_{x^{1} x^{1}}\left(x-h e_{2}\right)\right]_{+}-2\left[u_{x^{2} x^{2}}(x)\right]_{+}+w_{x^{2} x^{2}}^{h}(x) \\
& \geq \Delta w^{h}(x)-\left(w_{x^{1} x^{1}}^{h}(x)\right)_{-}+w_{x^{2} x^{2}}^{h}(x) .
\end{aligned}
$$

Thus, in $D_{h}^{-}, w^{h}$ is a viscosity supersolution of the uniformly elliptic equation $\Delta v-\left(v_{x^{1} x^{1}}\right)_{-}+v_{x^{2} x^{2}}=0$, with the same ellipticity constants $1,2 d$. Thus $w^{h}$ and hence $\Delta_{h, e_{2}}^{2} u \in \bar{S}(0)$ in $D_{h}^{-}$. Again, $u \in C^{2}(D)$ and $\bar{S}(0)$ is closed implies $u_{x^{2} x^{2}} \in \bar{S}(0)$ in $D^{-}$, and hence $\left(u_{x^{2} x^{2}}\right)_{-} \in \underline{S}(0)$ in $D$.

Proof of Theorem 1. We recall the $W^{2, \delta}$ interior estimate, due to Lin (see [Lin86], and Proposition 7.4 in [CC95]): if $u \in S(\lambda, \Lambda, 0)$ in $B_{r}$, there exists a universal $\delta>0$ such that $u \in W^{2, \delta}\left(B_{7 r / 8}\right)$ and

$$
\|u\|_{W^{2, \delta}\left(B_{7 r / 8}\right)} \leq C(d, \lambda, \Lambda) r^{-2+\frac{d}{\delta}} \sup _{B_{r}}|u| .
$$

This result, along with the well-known subsolution estimate (see Theorem 4.8 in CC95, Theorem 9.20 in GT83) (with $p=\delta$ ), applied to our subsolution $\left(u_{x^{1} x^{1}}\right)_{+}$ gives: $\forall B_{r}:=B_{r}\left(x_{0}\right) \subset \subset D$

$$
\begin{aligned}
\sup _{B_{3 r / 4}}\left(u_{x^{1} x^{1}}\right)_{+} & \leq N_{d}\left\{f_{B_{7 r / 8}}\left[\left(u_{x^{1} x^{1}}\right)_{+}\right]^{\delta} d x\right\}^{\frac{1}{\delta}}=N_{d}^{\prime} r^{-\frac{d}{\delta}}\left\|\left(u_{x^{1} x^{1}}\right)_{+}\right\|_{L^{\delta}\left(B_{7 r / 8}\right)} \\
& \leq N_{d}^{\prime} C r^{-2} \sup _{B_{r}}|u|
\end{aligned}
$$

with an identical estimate holding for $\left(u_{x^{2} x^{2}}\right)_{-}$. This and the inequality $|\Delta u(x)| \leq$ $\left(u_{x^{2} x^{2}}(x)\right)_{-}+\left(u_{x^{1} x^{1}}(x)\right)_{+}$immediately yield: $\forall B_{r}:=B_{r}\left(x_{0}\right) \subset \subset D$

$$
\sup _{B_{3 r / 4}}|\Delta u| \leq C_{d}^{\prime} r^{-2} \sup _{B_{r}}|u|,
$$

and hence $\forall 1<p<\infty$, the $W^{2, p}$ estimate in the form $\left\|u_{x x}\right\|_{L^{p}\left(B_{r / 2}\right)} \leq$ $C(d, p)\left\{r^{-2}\|u\|_{L^{p}\left(B_{3 r / 4}\right)}+\|\Delta u\|_{L^{p}\left(B_{3 r / 4}\right)}\right\}$ yields: $\forall B_{r}:=B_{r}\left(x_{0}\right) \subset \subset D$

$$
\left\|u_{x x}\right\|_{L^{p}\left(B_{r / 2}\right)} \leq C^{\prime \prime}(d, p) r^{-2+\frac{d}{p}} \sup _{B_{r}}|u| .
$$

The estimate for $0<p \leq 1$ follows from the estimate for $p=2$ and Hölder's inequality. Thus (1.2) holds $\forall 0<p<\infty$.

\section{Viscosity SOlutions of $\Delta u+\left(u_{x^{1} x^{1}}\right)_{+}-\left(u_{x^{2} x^{2}}\right)_{-}=0$}

Let $O \subset \mathbb{R}^{d}$ be open. Recall that a function $u \in C(O)$ is a viscosity subsolution of $F\left(u_{x x}\right)=0$ in $O$ if $\forall x_{0} \in O$ and $\varphi \in C^{2}(O)$, if $u-\varphi$ has a local maximum at $x_{0}$, then $F\left(\varphi_{x x}\left(x_{0}\right)\right) \geq 0$. Similarly, $u \in C(O)$ is a viscosity supersolution of $F\left(u_{x x}\right)=0$ in $O$ if $\forall x_{0} \in O$ and $\varphi \in C^{2}(O)$, if $u-\varphi$ has a local minimum at $x_{0}$, then $F\left(\varphi_{x x}\left(x_{0}\right)\right) \leq 0$. We say $u \in C(O)$ is a viscosity solution of $F\left(u_{x x}\right)=0$ in $O$ if $u$ is both a viscosity subsolution and supersolution in $O$.

If $u \in C(D)$ is a viscosity solution of $(0.1)$ in $D$, it is well known that $u$ is twice differentiable almost everywhere in $D$. That is, there exists a subset $E$ of 
full Lebesgue measure in $D$ such that $u$ is twice differentiable throughout $E$, and $\Delta u(x)+\left(u_{x^{1} x^{1}}(x)\right)_{+}-\left(u_{x^{2} x^{2}}(x)\right)_{-}=0 \forall x \in E$. Since $u$ is only assumed continuous in $D$, we know very little about the measurable sets $\left\{D: u_{x^{1} x^{1}}>0\right\}$ and $\left\{D: u_{x^{2} x^{2}}<0\right\}$. We consider the two important sets

$$
D^{+}:=\left\{D: \limsup _{h \rightarrow 0+} \Delta_{h, e_{1}}^{2} u>0\right\}, \quad D^{-}:=\left\{D: \liminf _{h \rightarrow 0+} \Delta_{h, e_{2}}^{2} u<0\right\},
$$

where for any unit vector $e \in \mathbb{R}^{d}$, and $h>0, \Delta_{h, e}^{2} u(x):=\frac{u(x+h e)+u(x-h e)-2 u(x)}{h^{2}}$.

The following lemma and theorem clarify the roles of $D^{+}$and $D^{-}$in the regularity of (0.1). In general, the interiors of $D^{+}, D^{-}$may be empty. However if their respective interiors are nonempty, the following lemma shows that $u$ will be $C^{2, \alpha}$ in the interior of these sets. In particular, if $D^{+}$is open, then $D^{+}=\left\{D: u_{x^{1} x^{1}}>0\right\}$.

Lemma 2.1. Let $u \in C(D)$ be a viscosity solution of $F\left(u_{x x}\right):=\Delta u+\left(u_{x^{1} x^{1}}\right)_{+}-$ $\left(u_{x^{2} x^{2}}\right)_{-}=0$ in $D$. If $O$ is any open set with either $O \subset D^{+}$or $O \subset D^{-}$, then $u \in C^{2, \alpha}(O)$.

Proof. Let $U$ be any open set with $U \subset D^{+}$. Then $u$ is a viscosity solution of the concave equation $F^{+}\left(u_{x x}\right):=\Delta u+u_{x^{1} x^{1}}-\left(u_{x^{2} x^{2}}\right)_{-}=0$ in $U$. To see this, fix $x_{0} \in U$ and $\varphi \in C^{2}(U)$ such that $u-\varphi$ has a local maximum at $x_{0}$. We claim that $F^{+}\left(\varphi_{x x}\left(x_{0}\right)\right) \geq 0$. By assumption, $0 \leq F\left(\varphi_{x x}\left(x_{0}\right)\right):=\Delta \varphi\left(x_{0}\right)+\left(\varphi_{x^{1} x^{1}}\left(x_{0}\right)\right)_{+}-$ $\left(\varphi_{x^{2} x^{2}}\left(x_{0}\right)\right)_{-}$. But for all $x$ in some $B_{r}\left(x_{0}\right) \subset U,(u-\varphi)(x) \leq(u-\varphi)\left(x_{0}\right)$. In particular, $\forall 0<h<r, \Delta_{h, e_{1}}^{2} u\left(x_{0}\right) \leq \Delta_{h, e_{1}}^{2} \varphi\left(x_{0}\right)$. Since $x_{0} \in D^{+}$and $\varphi$ is $C^{2}, 0<\lim _{\sup _{h \rightarrow 0+}} \Delta_{h, e_{1}}^{2} u\left(x_{0}\right) \leq \varphi_{x^{1} x^{1}}\left(x_{0}\right)$. Thus $0 \leq \Delta \varphi\left(x_{0}\right)+\varphi_{x^{1} x^{1}}\left(x_{0}\right)-$ $\left(\varphi_{x^{2} x^{2}}\left(x_{0}\right)\right)_{-}=F^{+}\left(\varphi_{x x}\left(x_{0}\right)\right)$, and hence $F^{+}\left(u_{x x}\right) \geq 0$ in $U$. But by the pde, $F^{+}\left(u_{x x}\right) \leq 0$ throughout $D$. Thus $F^{+}\left(u_{x x}\right)=0$ in $U$, and by the Evans-Krylov theorem, $u \in C^{2, \alpha}(U)$.

Similarly, if $V$ is any open set with $V \subset D^{-}$, then $u \in C^{2, \alpha}(V)$, since $u$ is a viscosity solution of the convex equation $G^{+}\left(u_{x x}\right):=\Delta u+\left(u_{x^{1} x^{1}}\right)_{+}+u_{x^{2} x^{2}}=0$ in $V$, and the result follows by the Evans-Krylov theorem. To see this, we need only show $G^{+}\left(u_{x x}\right) \leq 0$ in $V$, since by the pde, $G^{+}\left(u_{x x}\right) \geq 0$ throughout $D$. So fix $y_{0} \in V, \psi \in C^{2}(V)$ such that $u-\psi$ has a local minimum at $y_{0}$. We claim $G^{+}\left(\psi_{x x}\left(y_{0}\right)\right) \leq 0$. By assumption, $\Delta \psi\left(y_{0}\right)+\left(\psi_{x^{1} x^{1}}\left(y_{0}\right)\right)_{+}-\left(\psi_{x^{2} x^{2}}\left(y_{0}\right)\right)_{-}=$ $F\left(\psi_{x x}\left(y_{0}\right)\right) \leq 0$. But for all $y$ in some $B_{s}\left(y_{0}\right) \subset V,(u-\psi)\left(y_{0}\right) \leq(u-\psi)(y)$. In particular, $\forall 0<h<s, \Delta_{h, e_{2}}^{2} \psi\left(y_{0}\right) \leq \Delta_{h, e_{2}}^{2} u\left(y_{0}\right)$. Since $y_{0} \in D^{-}$and $\varphi$ is $C^{2}, \psi_{x^{2} x^{2}}\left(y_{0}\right) \leq \liminf _{h \rightarrow 0+} \Delta_{h, e_{2}}^{2} u\left(y_{0}\right)<0$. Thus $G^{+}\left(\psi_{x x}\left(y_{0}\right)\right)=\Delta \psi\left(y_{0}\right)+$ $\left(\psi_{x^{1} x^{1}}\left(y_{0}\right)\right)_{+}+\psi_{x^{2} x^{2}}\left(y_{0}\right) \leq 0$, and hence $G^{+}\left(u_{x x}\right) \leq 0$ in $V$.

Theorem 2. Let $u \in C(D)$ be a viscosity solution of $F\left(u_{x x}\right):=\Delta u+\left(u_{x^{1} x^{1}}\right)_{+}-$ $\left(u_{x^{2} x^{2}}\right)_{-}=0$ in $D$.

(i) If $D^{+}=\emptyset$ or $D^{+}=D$, then $u \in C^{2, \alpha}(D)$.

(ii) If $D^{+}$is not open, then $u \notin C^{2}\left(B_{r}\right)$, for any ball $B_{r} \subset D$ with $B_{r} \cap$ $\left[D^{+} \backslash \operatorname{int}\left(D^{+}\right)\right] \neq \emptyset$.

(iii) If int $\left(D^{+}\right) \neq \emptyset$, then $u \in C_{\text {loc }}^{2, \alpha}\left(\right.$ int $\left.\left(D^{+}\right)\right)$.

(iv) If $D^{+}$and $D^{-}$are both open, $u_{x^{1} x^{1}}$ is continuous on $\partial D^{+} \cap D$, and $u_{x^{2} x^{2}}$ is continuous on $\partial D^{-} \cap D$, then $u \in W_{\text {loc }}^{2, p}(D) \forall 0<p<\infty$, and $\forall B_{r} \subset \subset D$, $\left\|u_{x x}\right\|_{L^{p}\left(B_{r / 2}\right)} \leq C(d, p) r^{-2+\frac{d}{p}} \sup _{B_{r}}|u|$.

In (i)-(iii), corresponding statements hold for $D^{-}$. 
Proof. There are precisely 5 cases for $D^{+}$.

Case 1. If $D^{+}=\emptyset$, then $\lim \sup _{h \rightarrow 0+} \Delta_{h, e_{1}}^{2} u(x) \leq 0, \forall x \in D$. In particular, $u_{x^{1} x^{1}}(x) \leq 0 \forall x \in E$, and thus $F^{-}\left(u_{x x}\right):=\Delta u-\left(u_{x^{2} x^{2}}\right)_{-}=0$ a.e. in $D$, from which it follows that $\forall B_{r}:=B_{r}(y) \subset \subset D, u \in C^{2, \alpha}\left(B_{r}\right)$. To see this, fix $B_{r} \subset \subset D$, and let $v$ be the $C^{2, \alpha}\left(B_{r}\right) \cap C\left(\bar{B}_{r}\right)$ solution of

$$
\left\{\begin{aligned}
F^{-}\left(v_{x x}\right):=\Delta v-\left(v_{x^{2} x^{2}}\right)_{-} & =0 \\
v & \text { in } B_{r} \\
& \text { on } \partial B_{r} .
\end{aligned}\right.
$$

Then $u-v \in S\left(F^{-}\left(u_{x x}\right)\right):=S\left(\frac{1}{d}, 2 d, F^{-}\left(u_{x x}\right)\right)$ in $B_{r}$, and since $F^{-}\left(u_{x x}\right)=0$ a.e. in $B_{r}$, the $\mathrm{ABP}$ estimate yields

$$
\sup _{B_{r}}|u-v| \leq \sup _{\partial B_{r}}|u-v|+N_{d} r\left\|F^{-}\left(u_{x x}\right)\right\|_{L^{d}\left(B_{r}\right)}=0 .
$$

Thus $u=v \in C^{2, \alpha}\left(B_{r}\right)$. That is, $u$ is a $C^{2, \alpha}(D)$ solution of $F^{-}\left(u_{x x}\right)=0$ in $D$. Similarly, if $D^{-}=\emptyset, u$ is a $C^{2, \alpha}(D)$ solution of $G^{-}\left(u_{x x}\right)=0$ in $D$. Here, we've used the class $S\left(\frac{1}{d}, 2 d, f\right)$ with bounded measurable $f$. See remark 2.2.3 in [CC95].

Case 2. If $D^{+}=D$, then by Lemma $2.1, u$ is a $C^{2, \alpha}(D)$ solution of $F^{+}\left(u_{x x}\right)=0$ in $D$, while if $D^{-}=D, u$ is a $C^{2, \alpha}(D)$ solution of $G^{+}\left(u_{x x}\right)=0$ in $D$.

Case 3. If $\operatorname{int}\left(D^{+}\right)=\emptyset, D^{+} \neq \emptyset$, i.e. $D^{+}=D^{+} \backslash \operatorname{int}\left(D^{+}\right)$, then $u_{x^{1} x^{1}}$ is discontin-

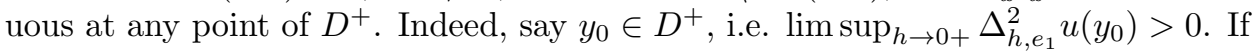
$u_{x^{1} x^{1}}\left(y_{0}\right)$ doesn't exist, the conclusion trivially holds. If $u_{x^{1} x^{1}}\left(y_{0}\right)$ does exist, then $u_{x^{1} x^{1}}\left(y_{0}\right)>0$, but since $y_{0} \notin \operatorname{int}\left(D^{+}\right)$, then $\forall \varepsilon>0, B_{\varepsilon}\left(y_{0}\right) \nsubseteq D^{+}$. Thus $\exists\left\{z_{n}\right\}$ such that $z_{n} \rightarrow y_{0}$ but $\lim \sup _{h \rightarrow 0+} \Delta_{h, e_{1}}^{2} u\left(z_{n}\right) \leq 0$, hence $u_{x^{1} x^{1}}$ is discontinuous at $y_{0}$. In this case, $u \notin C^{2}\left(B_{r}\right)$ for any ball with $B_{r} \cap D^{+} \neq \emptyset$. Similarly, if $\operatorname{int}\left(D^{-}\right)=\emptyset, D^{-} \neq \emptyset$, then $u \notin C^{2}\left(B_{r}\right)$ for any ball with $B_{r} \cap D^{-} \neq \emptyset$.

Case 4. If $\operatorname{int}\left(D^{+}\right)=D^{+}$, then by Lemma 2.1, $F^{+}\left(u_{x x}\right)=0$ throughout $D^{+}$, $u \in C^{2, \alpha}\left(D^{+}\right)$, and $D^{+}=\left\{D, u_{x^{1} x^{1}}>0\right\}$. For $h>0$, define $D_{h}^{+}:=\left\{x \in D^{+}\right.$: $\left.\operatorname{dist}\left(x, \partial D^{+}\right)>h\right\}$. Fix $x \in D_{h}^{+}$and set $w^{h}(x)=u\left(x+h e_{1}\right)+u\left(x-h e_{1}\right)-$ $2 u(x)$. Since $F^{+}\left(u_{x x}\left(x \pm h e_{1}\right)\right)=0$ in $D_{h}^{+}$, the exact argument used in Lemma 1.1 implies $u_{x_{1} x_{1}} \in \underline{S}(0)$ in $D^{+}$. There are two possibilities. Either (a) $\exists x_{0} \in$ $D_{0}^{+}:=\left\{D, \lim \sup _{h \rightarrow 0+} \Delta_{h, e_{1}}^{2} u=0\right\}$, where $u_{x^{1} x^{1}}$ is discontinuous; or (b) $u_{x^{1} x^{1}}$ is continuous in $D_{0}^{+}=\left\{D, u_{x^{1} x^{1}}=0\right\}$. If (b) holds, then $u_{x^{1} x^{1}} \in C\left(\overline{D^{+}}\right)$, and since $u_{x^{1} x^{1}} \in \underline{S}(0)$ in $D^{+}$, it follows that $\left(u_{x^{1} x^{1}}\right)_{+} \in \underline{S}(0)$ in $D$. Hence, exactly as in the proof of Theorem 1, the $W^{2, \delta}$ interior estimate, along with the subsolution estimate (with $p=\delta$ ), applied to our subsolution $\left(u_{x^{1} x^{1}}\right)+$ gives: $\forall B_{r} \subset \subset D$

$$
\sup _{B_{3 r / 4}}\left(u_{x^{1} x^{1}}\right)_{+} \leq N_{d}^{\prime} r^{-2} \sup _{B_{r}}|u| .
$$

If also $\operatorname{int}\left(D^{-}\right)=D^{-}$, then $G^{+}\left(u_{x x}\right)=0$ throughout $D^{-}, u \in C^{2, \alpha}\left(D^{-}\right)$, and $D^{-}=\left\{D, u_{x^{2} x^{2}}<0\right\}$. An analogous argument to the above shows that $u_{x_{2} x_{2}} \in$ $\bar{S}(0)$ in $D^{-}$. Again, either $(a)^{\prime} \exists x_{0} \in D_{0}^{-}:=\left\{D, \liminf _{h \rightarrow 0+} \Delta_{h, e_{2}}^{2} u=0\right\}$, where $u_{x^{2} x^{2}}$ is discontinuous; or $(b)^{\prime} u_{x^{2} x^{2}}$ is continuous in $D_{0}^{-}=\left\{D, u_{x^{2} x^{2}}=0\right\}$. If $(b)^{\prime}$ holds, then $u_{x^{2} x^{2}} \in C\left(\overline{D^{-}}\right)$, and since $u_{x_{2} x_{2}} \in \bar{S}(0)$ in $D^{-}$, it follows that $\left(u_{x^{2} x^{2}}\right)_{-} \in \underline{S}(0)$ in $D$. Hence, the $W^{2, \delta}$ interior estimate in conjunction with the subsolution estimate applied to $\left(u_{x^{2} x^{2}}\right)$ - gives: $\forall B_{r} \subset \subset D$

$$
\sup _{B_{3 r / 4}}\left(u_{x^{2} x^{2}}\right)_{-} \leq N_{d}^{\prime \prime} r^{-2} \sup _{B_{r}}|u| .
$$


Inequalities (2.2), (2.3) and the inequality $|\Delta u(x)| \leq\left(u_{x^{2} x^{2}}(x)\right)_{-}+\left(u_{x^{1} x^{1}}(x)\right)_{+}$, which holds a.e. in $D$, immediately give: $\forall B_{r} \subset \subset D$

$$
\|\Delta u\|_{L^{\infty}\left(B_{3 r / 4}\right)} \leq C_{d}^{\prime} r^{-2} \sup _{B_{r}}|u|
$$

and hence $\forall 1<p<\infty$, the $W^{2, p}$ inequality in the form $\left\|u_{x x}\right\|_{L^{p}\left(B_{r / 2}\right)} \leq$ $C(d, p)\left\{r^{-2}\|u\|_{L^{p}\left(B_{3 r / 4}\right)}+\|\Delta u\|_{L^{p}\left(B_{3 r / 4}\right)}\right\}$ yields: $\forall B_{r} \subset \subset D$

$$
\left\|u_{x x}\right\|_{L^{p}\left(B_{r / 2}\right)} \leq C^{\prime \prime}(d, p) r^{-2+\frac{d}{p}} \sup _{B_{r}}|u| .
$$

The estimate for $0<p \leq 1$ follows from the estimate for $p=2$ and Hölder's inequality.

Case 5. Finally, if $\operatorname{int}\left(D^{+}\right) \neq D^{+}, D, \emptyset$, then $D^{+} \backslash \operatorname{int}\left(D^{+}\right) \neq \emptyset$, and as in Case $3, u \notin C^{2}\left(B_{r}\right)$, for any ball $B_{r} \subset D$ such that $B_{r} \cap\left[D^{+} \backslash \operatorname{int}\left(D^{+}\right)\right] \neq \emptyset$. The same statement, but with $D^{-}$in place of $D^{+}$, also holds. On the other hand, since $\operatorname{int}\left(D^{+}\right) \neq \emptyset, \exists O \subset D^{+}$open. By Lemma 2.1, $u \in C^{2, \alpha}(O)$. Simliarly, if $\operatorname{int}\left(D^{-}\right) \neq \emptyset, \exists V \subset D^{-}$open and by Lemma $2.1, u \in C^{2, \alpha}(V)$.

Remark. Theorem 2 (iv) implies, in particular, that $C^{2}(D)$ solutions of $F\left(u_{x x}\right)=0$ in $D$ belong to $W_{l o c}^{2, p}(D), \forall 0<p<\infty$, as proved in Theorem 1. Thus Theorem 2 is a slight generalization of our result in [Kov12. In Case 4 (a), i.e. $\operatorname{int}\left(D^{+}\right)=D^{+}$, but $u_{x^{1} x^{1}}$ is discontinuous somewhere in $D_{0}^{+}$, the Evans-Krylov theorem ensures that the $C_{l o c}^{2, \alpha}\left(D^{+}\right)$estimates hold inside $D^{+}$. In particular, the following $W_{l o c}^{2, \infty}\left(D^{+}\right)$estimate holds: $\forall B_{r} \subset \subset D^{+}, \sup _{B_{r / 2}}\left\|u_{x x}\right\| \leq C_{d} r^{-2} \sup _{B_{r}}|u|$. The same estimate holds inside the open set $D^{-}$in case $4(a)^{\prime}$. More generally, if $\operatorname{int}\left(D^{+}\right) \neq \emptyset$, then by Theorem 2 (iii), these same estimates hold inside of any ball $B_{r} \subset \subset O$, where $O \subseteq D^{+}$. An identical statement holds for balls $B_{r} \subset \subset V$, where $V \subseteq D^{-}$, in case $\operatorname{int}\left(D^{-}\right) \neq \emptyset$.

The obvious question remains: does the $W^{2, p}$ estimate (2.4) hold if neither $D^{+}$, nor $D^{-}$is open? Say $B_{r} \subset \subset D$. By the standard $W^{2, p}$ inequality, if we can show

$$
\|\Delta u\|_{L^{\infty}\left(B_{3 r / 4}\right)} \leq C_{d} r^{-2} \sup _{B_{r}}|u|,
$$

then estimate (2.4) $(1<p<\infty)$ follows. It is possible that estimate (2.4) holds for $p>1$ even if (2.5) fails, e.g. if $\Delta u \in L^{p}\left(B_{3 r / 4}\right)$. Or, it might be the case that viscosity solutions of $(0.1)$ belong to $W_{l o c}^{2, p_{0}}(D)$ for some $p_{0}>1$ but not to $W_{l o c}^{2, q}(D)$ for $q>p_{0}$. This question remains open.

Moreover, it seems entirely possible that viscosity solutions $u \in C(D)$ of $(0.1)$ can be $C^{2}$ nowhere in $D$, that is, $u \notin C^{2}(O)$, for any open set $O \subset D$. If so, this would imply that none of the 4 equations $F^{ \pm}\left(u_{x x}\right)=0, G^{ \pm}\left(u_{x x}\right)=0$ holds anywhere in $D$ (i.e. in any open set), and thus, by Lemma 2.1 , both $D^{+}$and $D^{-}$ have empty interiors in $D$. More precisely, it would imply neither of $D^{+}$or $D^{-}$is empty, and every point of each is a noninterior point. This question is open.

One thing we can say for certain is that if $O \subset D$ is open and $u \in C^{2}(O)$, then one of $F^{+}\left(u_{x x}\right)=0$ or $F^{-}\left(u_{x x}\right)=0$ holds somewhere in $O$ (with a corresponding statement for $G^{+}, G^{-}$). More precisely, we have the following necessary condition for solutions being $C^{2}$ in an open set in $D$. 
Proposition 2.2. Let $u \in C(D)$ be a viscosity solution of $F\left(u_{x x}\right):=\Delta u+$ $\left(u_{x^{1} x^{1}}\right)_{+}-\left(u_{x^{2} x^{2}}\right)_{-}=0$ in $D$. If $O \subset D$ is open and $u \in C^{2}(O)$, then $\exists V \subset O$ (open) such that one of $F^{+}\left(u_{x x}\right)=0$ or $F^{-}\left(u_{x x}\right)=0$ holds in $V$ (with a corresponding result for $\left.G^{+}, G^{-}\right)$.

Proof. If not, then $\forall V \subset O$ open, $F^{+}\left(u_{x x}\right) \nsupseteq 0$ in $V$ and $F^{-}\left(u_{x x}\right) \nsupseteq 0$ in $V$. In particular, this implies $D^{+}$has no interior points in $O$. Since if $\exists x_{0} \in O$ that is an interior point of $D^{+}$, i.e. $\exists \varepsilon>0$ with $B_{\varepsilon}\left(x_{0}\right) \subset D^{+}$(and since $x_{0} \in O$ (open), then we can always take $\varepsilon>0$ so small as to ensure $\left.B_{\varepsilon}\left(x_{0}\right) \subset O\right)$, then by Lemma 2.1, this implies $F^{+}\left(u_{x x}\right)=0$ in $B_{\varepsilon}\left(x_{0}\right)$, a contradiction. But if $D^{+}$has no interior points in $O$, then either: (a) $O \cap D^{+}=\emptyset$, or (b) $\left(O \cap D^{+}\right) \backslash \operatorname{int}\left(D^{+}\right) \neq \emptyset$. If (a) holds, then every $x \in O$ satisfies $\limsup _{h \rightarrow 0+} \Delta_{h, e_{1}}^{2} u(x) \leq 0$. Since $u \in C^{2}(O)$, this implies $u_{x^{1} x^{1}} \leq 0$ in $O$, and hence $F^{-}\left(u_{x x}\right)=0$ in $O$, a contradiction. If (b) holds, then $u_{x^{1} x^{1}}$ will be discontinuous at any point of $\left(O \cap D^{+}\right) \backslash \operatorname{int}\left(D^{+}\right)$, which contradicts the assumption $u \in C^{2}(O)$.

We close this section with a lemma which gives a weaker sufficient condition for the continuity of the second derivatives than that stated in Lemma 2.1. Its proof uses the Aleksandrov-Bakelman-Pucci (ABP) estimate.

Lemma 2.3. Let $u \in C(D)$ be a viscosity solution of $F\left(D^{2} u\right):=\Delta u+\left(u_{x^{1} x^{1}}\right)_{+}-$ $\left(u_{x^{2} x^{2}}\right)_{-}=0$ in $D$. If $O \subset D$ is any open set such that either $u_{x^{1} x^{1}} \geq 0$ holds a.e. in $O$ or $u_{x^{2} x^{2}} \leq 0$ holds a.e. in $O$, then $u \in C^{2, \alpha}(O)$.

Proof. Say $u_{x^{1} x^{1}} \geq 0$ a.e. in $O$. Fix $B_{r}:=B_{r}(y) \subset \subset O$ and let $w$ be the $C^{2, \alpha}\left(B_{r}\right) \cap$ $C\left(\bar{B}_{r}\right)$ solution of

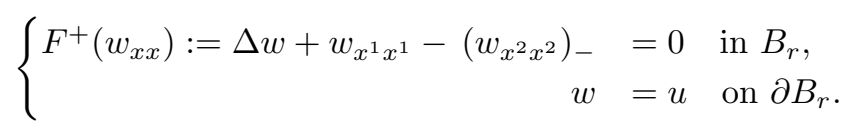

Since $F^{+}\left(u_{x x}\right) \leq 0=F^{+}\left(w_{x x}\right)$ in $B_{r}, u-w \in \bar{S}(0):=\bar{S}\left(\frac{1}{d}, 2 d, 0\right)$ in $B_{r}$, and by the maximum principle, $w \leq u$ in $\overline{B_{r}}$. Also $u-w \in \underline{S}\left(-F\left(w_{x x}\right)\right)=\underline{S}\left(-\left(w_{x^{1} x^{1}}\right)_{-}\right)$ in $B_{r}$, and by the ABP estimate

$$
\sup _{B_{r}}(u-w) \leq \sup _{\partial B_{r}}(u-w)+N_{d} r\left\|\left(w_{x^{1} x^{1}}\right)_{-}\right\|_{L^{d}\left(\Gamma^{+}\right)}=N_{d} r\left\|\left(w_{x^{1} x^{1}}\right)_{-}\right\|_{L^{d}\left(\Gamma^{+}\right)},
$$

where $\Gamma^{+}$denotes the upper contact set of $u-w$ in $B_{r}$. In $\Gamma^{+}, u-w$ is concave, and hence for almost every $x \in \Gamma^{+}, u_{x x}(x) \leq w_{x x}(x)$. In particular, $u_{x^{1} x^{1}} \leq w_{x^{1} x^{1}}$ a.e. in $\Gamma^{+}$, and hence $\left(w_{x^{1} x^{1}}\right)_{-} \leq\left(u_{x^{1} x^{1}}\right)_{-}=0$ a.e. in $\Gamma^{+}$. Hence $\forall x \in \bar{B}_{r}, 0 \leq$ $u(x)-w(x) \leq N_{d} r\left\|\left(w_{x^{1} x^{1}}\right)_{-}\right\|_{L^{d}\left(\Gamma^{+}\right)}=0$. Thus $u=w \in C^{2, \alpha}\left(B_{r}\right)$.

Similarly, if $u_{x^{2} x^{2}} \leq 0$ holds a.e. in $O$, let $v$ be the $C^{2, \alpha}\left(B_{r}\right) \cap C\left(\bar{B}_{r}\right)$ solution of

$$
\left\{\begin{aligned}
G^{+}\left(v_{x x}\right):=\Delta v+\left(v_{x^{1} x^{1}}\right)_{+}+v_{x^{2} x^{2}} & =0 & & \text { in } B_{r} \\
v & =u & & \text { on } \partial B_{r} .
\end{aligned}\right.
$$

By the maximum principle, $u \leq v$ in $\bar{B}_{r}$. Also $u-v \in \bar{S}\left(-F\left(v_{x x}\right)\right)=\bar{S}\left(\left(v_{x^{2} x^{2}}\right)_{+}\right)$ in $B_{r}$, and by the ABP estimate

$$
\inf _{B_{r}}(u-v) \geq \inf _{\partial B_{r}}(u-v)-N_{d} r\left\|\left(v_{x^{2} x^{2}}\right)_{+}\right\|_{L^{d}\left(\Gamma^{-}\right)}=-N_{d} r\left\|\left(v_{x^{2} x^{2}}\right)_{+}\right\|_{L^{d}\left(\Gamma^{-}\right)},
$$

where $\Gamma^{-}$denotes the lower contact set of $u-v$ in $B_{r}$. In $\Gamma^{-}, u-v$ is convex, and hence for almost every $x \in \Gamma^{-}, v_{x x}(x) \leq u_{x x}(x)$, from which it follows 
that $\left(v_{x^{2} x^{2}}\right)_{+} \leq\left(u_{x^{2} x^{2}}\right)_{+}=0$ a.e. in $\Gamma^{-}$. Hence $\forall x \in \bar{B}_{r}, 0 \leq v(x)-u(x) \leq$ $N_{d} r\left\|\left(v_{x^{2} x^{2}}\right)_{+}\right\|_{L^{d}\left(\Gamma^{-}\right)}=0$, and hence $u=v \in C^{2, \alpha}\left(B_{r}\right)$.

\section{REFERENCES}

[CC95] Luis A. Caffarelli and Xavier Cabré, Fully nonlinear elliptic equations, American Mathematical Society Colloquium Publications, vol. 43, American Mathematical Society, Providence, RI, 1995. MR.1351007 (96h:35046)

[CC03] Xavier Cabré and Luis A. Caffarelli, Interior $C^{2, \alpha}$ regularity theory for a class of nonconvex fully nonlinear elliptic equations (English, with English and French summaries), J. Math. Pures Appl. (9) 82 (2003), no. 5, 573-612, DOI 10.1016/S0021-7824(03)000291. MR.1995493 (2004f:35049)

[CY00] Luis A. Caffarelli and Yu Yuan, A priori estimates for solutions of fully nonlinear equations with convex level set, Indiana Univ. Math. J. 49 (2000), no. 2, 681-695, DOI 10.1512/iumj.2000.49.1901. MR.1793687 (2002b:35049)

[Eva82] Lawrence C. Evans, Classical solutions of fully nonlinear, convex, second-order elliptic equations, Comm. Pure Appl. Math. 35 (1982), no. 3, 333-363, DOI 10.1002/cpa.3160350303. MR649348 (83g:35038)

[FN93] W. H. Fleming and M. Nisio, Differential games for stochastic partial differential equations, Nagoya Math. J. 131 (1993), 75-107. MR.1238634 (94h:93074)

[FS89] W. H. Fleming and P. E. Souganidis, On the existence of value functions of two-player, zero-sum stochastic differential games, Indiana Univ. Math. J. 38 (1989), no. 2, 293-314, DOI 10.1512/iumj.1989.38.38015. MR997385(90e:93089)

[GT83] David Gilbarg and Neil S. Trudinger, Elliptic partial differential equations of second order, 2nd ed., Grundlehren der Mathematischen Wissenschaften [Fundamental Principles of Mathematical Sciences], vol. 224, Springer-Verlag, Berlin, 1983. MR737190 (86c:35035)

[Ish89] Hitoshi Ishii, On uniqueness and existence of viscosity solutions of fully nonlinear second-order elliptic PDEs, Comm. Pure Appl. Math. 42 (1989), no. 1, 15-45, DOI 10.1002/cpa.3160420103. MR 973743 (89m:35070)

[Kov09] Jay Kovats, Value functions and the Dirichlet problem for Isaacs equation in a smooth domain, Trans. Amer. Math. Soc. 361 (2009), no. 8, 4045-4076, DOI 10.1090/S00029947-09-04732-1. MR2500878 (2010c:49057)

[Kov12] Jay Kovats, The minmax principle and $W^{2, p}$ regularity for solutions of the simplest Isaacs equations, Proc. Amer. Math. Soc. 140 (2012), no. 8, 2803-2815, DOI 10.1090/S0002-9939-2012-11610-7. MR2910767

[Kry83] N. V. Krylov, Boundedly inhomogeneous elliptic and parabolic equations in a domain (Russian), Izv. Akad. Nauk SSSR Ser. Mat. 47 (1983), no. 1, 75-108. MR688919 (85g:35046)

[Kry14a] N. V. Krylov, On the dynamic programming principle for uniformly nondegenerate stochastic differential games in domains and the Isaacs equations, Probab. Theory Related Fields 158 (2014), no. 3-4, 751-783, DOI 10.1007/s00440-013-0495-y. MR3176364

[Kry14b] N. V. Krylov, On regularity properties and approximations of value functions for stochastic differential games in domains, Ann. Probab. 42 (2014), no. 5, 2161-2196, DOI 10.1214/13-AOP848. MR 3262500

[Lin86] Fang-Hua Lin, Second derivative $L^{p}$-estimates for elliptic equations of nondivergent type, Proc. Amer. Math. Soc. 96 (1986), no. 3, 447-451, DOI 10.2307/2046592. MR822437 (88b:35058)

[Nis88] Makiko Nisio, Stochastic differential games and viscosity solutions of Isaacs equations, Nagoya Math. J. 110 (1988), 163-184. MR945913 (90b:93100)

[NV07] Nikolai Nadirashvili and Serge Vlăduț, Nonclassical solutions of fully nonlinear elliptic equations, Geom. Funct. Anal. 17 (2007), no. 4, 1283-1296, DOI 10.1007/s00039-0070626-7. MR 2373018 (2008m:35121)

[NV08] Nikolai Nadirashvili and Serge Vlăduț, Singular viscosity solutions to fully nonlinear elliptic equations (English, with English and French summaries), J. Math. Pures Appl. (9) 89 (2008), no. 2, 107-113, DOI 10.1016/j.matpur.2007.10.004. MR.2391642 (2009a:35080) 
[NV11] Nikolai Nadirashvili and Serge Vlăduţ, Singular solutions of Hessian fully nonlinear elliptic equations, Adv. Math. 228 (2011), no. 3, 1718-1741, DOI 10.1016/j.aim.2011.06.030. MR2824567(2012h:35077)

[Roc70] R. Tyrrell Rockafellar, Convex analysis, Princeton Mathematical Series, No. 28, Princeton University Press, Princeton, N.J., 1970. MR0274683 (43 \#445)

[Tru89] Neil S. Trudinger, On the twice differentiability of viscosity solutions of nonlinear elliptic equations, Bull. Austral. Math. Soc. 39 (1989), no. 3, 443-447, DOI 10.1017/S0004972700003361. MR995142 (90f:35038)

[Yua01] Yu Yuan, A priori estimates for solutions of fully nonlinear special Lagrangian equations (English, with English and French summaries), Ann. Inst. H. Poincaré Anal. Non Linéaire 18 (2001), no. 2, 261-270, DOI 10.1016/S0294-1449(00)00065-2. MR1808031 (2002e:35088)

Department of Mathematical Sciences, Florida Institute of Technology, Melbourne, FLORIDA 32901

E-mail address: jkovats@fit.edu 